\title{
Strangeness Suppression and Color Deconfinement ${ }^{\star}$
}

\author{
Helmut Satz ${ }^{1, \star \star}$ \\ ${ }^{1}$ Fakultät für Physik, Universität Bielefeld, Germany
}

\begin{abstract}
The relative multiplicities for hadron production in different high energy collisions are in general well described by an ideal gas of all hadronic resonances, except that under certain conditions, strange particle rates are systematically reduced. We show that the suppression factor $\gamma_{s}$, accounting for reduced strange particle rates in $p p, p A$ and $A A$ collisions at different collision energies, becomes a universal function when expressed in terms of the initial entropy density $s_{0}$ or the initial temperature $T$ of the produced thermal medium. It is found that $\gamma_{s}$ increases from about 0.5 to 1.0 in a narrow temperature range around the quark-hadron transition temperature $T_{c} \simeq 160 \mathrm{MeV}$. Strangeness suppression thus disappears with the onset of color deconfinement; subsequently, full equilibrium resonance gas behavior is attained.
\end{abstract}

The relation of color deconfinement and strange hadron production in high energy collisions has attracted much attention over more than thirty years. It had been observed that in elementary $\left(p p, e^{+} e^{-}\right)$collisions as well as in low energy nucleus-nucleus interactions, the production of strange particles was suppressed relative to the rates expected for an equilibrium resonance gas. In this context, it was argued early on that quark gluon plasma formation would enhance strange particle production in nucleus-nucleus collisions [1,2], bringing the produced system closer to equilibrium resonance gas rates. Over the years, different mechanisms for such an effect were discussed [3-6, 8]. The aim of the present paper is to provide clear experimental evidence showing that in $A A, p A$ and $p p$ collisions, strangeness production as function of the initial temperature of the produced thermal medium follows a universal form, with suppression ending in a narrow temperature band around the color deconfinement temperature.

High energy proton-proton, proton-nucleus and nucleus-nucleus collisions lead to abundant multihadron production. The relative rates of the secondaries thus produced are well accounted for by an ideal gas of all hadrons and hadronic resonances at fixed temperature $T$ and baryochemical potential $\mu$, with one well-known caveat. Strangeness production is reduced with respect to the rates thus predicted. This suppression can, however, be taken into account by one further parameter, $0<\gamma_{s} \leq 1$, if the predicted rate for a hadron species containing $v=1,2,3$ strange quarks is suppressed by the factor $\gamma_{s}^{v}[7]$.

The basic quantity for the resonance gas description is the grand-canonical partition function for an ideal gas at temperature $T$ in a spatial volume $V$

$$
\ln Z(T)=V \sum_{i} \frac{d_{i} \gamma_{s}^{\nu_{i}}}{(2 \pi)^{3}} \phi\left(m_{i}, T\right),
$$

\footnotetext{
${ }^{\star}$ based on joint work [arXiv:1709.02706] with P. Castorina and S. Plumari, U. di Catania, Italy

$\star \star$ e-mail: satz@physik.uni-bielefeld.de
} 
with $d_{i}$ specifying the degeneracy (spin, isospin) of species $i$, and $m_{i}$ its mass; the sum runs over all species. For simplicity, we assume for the moment that the baryochemical potential vanishes, $\mu=0$. In eq. (1)

$$
\phi\left(m_{i}, T\right)=\int d^{3} p \exp \left\{\sqrt{p^{2}+m_{i}^{2}} / T\right\} \sim \exp -\left(m_{i} / T\right)
$$

is the Boltzmann factor for species $i$, so that the ratio of the production rates $N_{i}$ and $N_{j}$ for hadrons of species $i$ and $j$ is given by

$$
\frac{N_{i}}{N_{j}}=\frac{d_{i} \gamma_{s}^{\gamma_{i}} \phi\left(m_{i}, T\right)}{d_{j} \gamma_{s}^{\gamma_{j}} \phi\left(m_{j}, T\right)},
$$

where $v_{i}=0,1,2,3$ specifies the number of strange quarks in species $i$. We note that in the grandcanonical formulation the volume cancels out in the form for the relative abundances.

Both temperature $T$ and strangeness suppression factor $\gamma_{s}$ have been measured at various collision energies and for different collision configurations. The resulting temperature of the emerging resonance gas is found to have a universal value $T_{c} \simeq 160 \pm 10 \mathrm{MeV}$ for all (high) collision energies and all collision configurations, see Fig. 1.

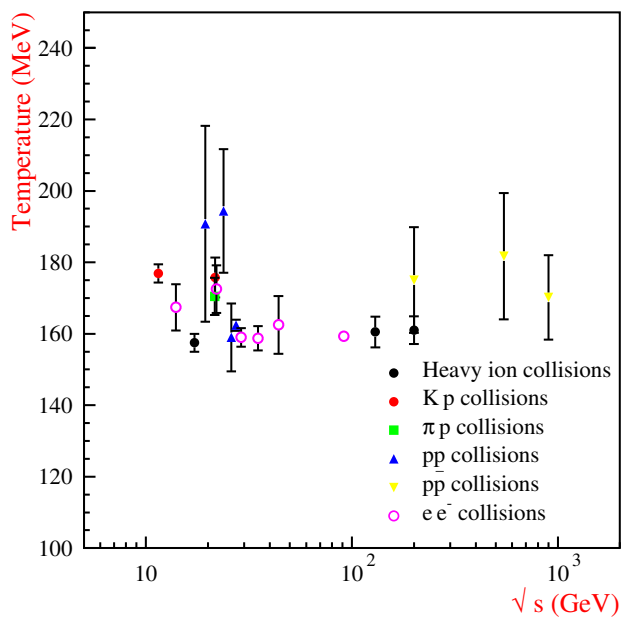

Figure 1. Hadronization temperatures for different collision energies and different collision configurations [9].

In contrast, the strangeness suppression factor $\gamma_{s}$ depends over a wide range on the collision energy and configuration; in particular, it differs considerably in $p p$ and $A A$ interactions, see Fig. 2. We note here that for $p p$ data, strangeness conservation generally is generally taken into account exactly, rather than in a grand canonical scheme with a strangeness chemical potential.

The curves in Fig. 2 are interpolation fits, leading to

$$
\gamma_{s}^{p}(s)=1-c_{p} \exp \left(-d_{p} s^{1 / 4}\right),
$$

for $p p$ and

$$
\gamma_{s}^{A}(s)=1-c_{A} \exp \left(-d_{A} \sqrt{A \sqrt{s}}\right)
$$




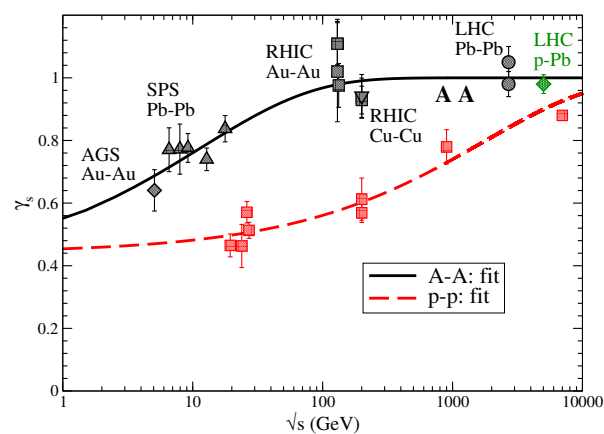

Figure 2. Strangeness suppression in $p p$ and $A A$ collisions at cms energy $\sqrt{s}[10]$.

for $A A$ collisions, with $c_{p}=0.5595 ; d_{p}=0.0242 ; c_{A}=0.606, d_{A}=0.0209$. It is evident from these fits that for sufficiently high collision energies, one expects $\gamma_{s}$ to converge to unity for $p p$ as well as for $A A$ collisions, so that strangeness suppression then also disappears in proton-proton interactions. This restoration of an equilibrium grand-canonical strangeness distribution has also been suggested on theoretical grounds [8].

The results shown in Fig. 2 suggest an obvious question: is there a unified description for the strangeness suppression observed in $A A$ and as well as in $p p$ collisions? Our aim here is to that if we consider the collision energy $\sqrt{s}$ by more natural thermal variables, then $\gamma_{s}$ in fact becomes one universal function.

The canonical view of a high energy collision is shown in Fig. 3. At proper time $\tau_{0}$, a thermal medium is formed, which at proper time $\tau_{h}$ hadronizes, i.e., freezes out into an ideal resonance gas.

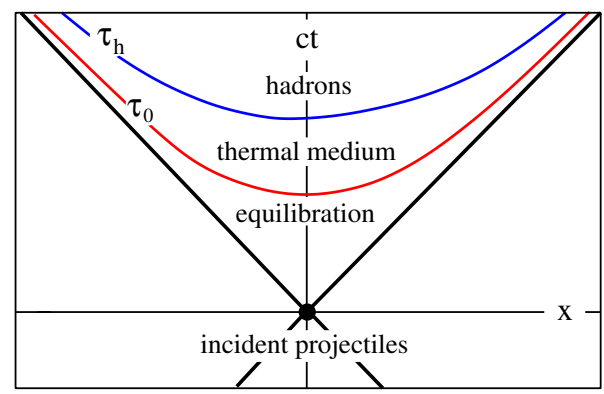

Figure 3. High energy collision in terms of longitudinal space $x$ and time $t$ in the center of mass.

The initial entropy density $s_{0}$ at the thermalisation time $\tau_{0}$ is for one-dimensional hydrodynamic expansion given by the Bjorken relation

$$
s_{0} \tau_{0} \simeq \frac{1.5 A^{x}}{\pi R_{x}^{2}}\left(\frac{d N}{d y}\right)_{y=0}^{x}, \text { with } x \sim p p, p A, A A,
$$

where $(d N / d y)^{x}(s)$ denotes the average multiplicity per unit central rapidity in the corresponding reaction $x$ at collision energy $\sqrt{s}$, while $R_{x}$ gives the radius of the associated transverse area. This multiplicity has been measured at different collision energies for $p p$ as well as for $A A$ collisions, with the result shown in Fig. 4

Using $\left(d N_{c h} / d \eta\right) / 0.5\left\langle N_{\text {part }}\right\rangle$ for $(d N / d y)_{y=0}$, the fit curves shown in this figure are given by 


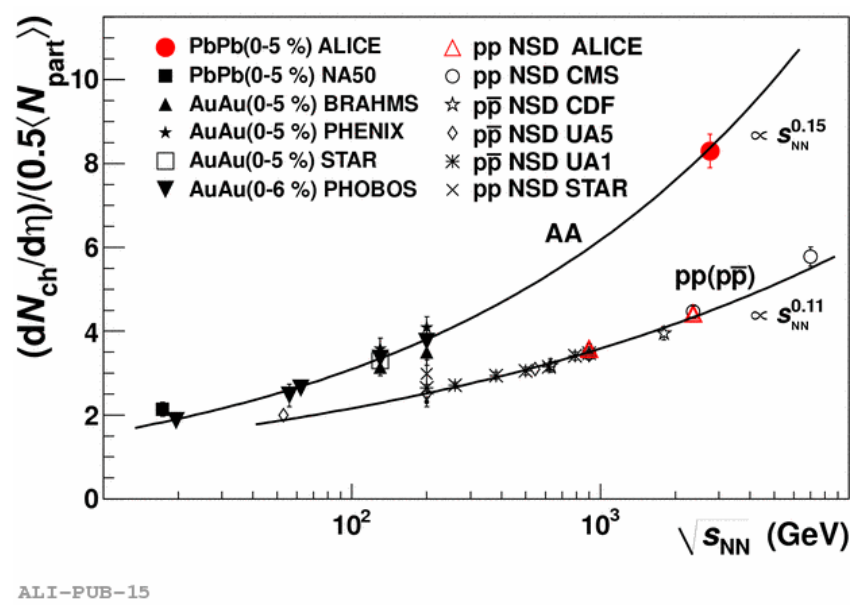

Figure 4. Average multiplicities in $p p$ and $A A$ collisions as function of cms energy $\sqrt{s}$ [11].

$$
\left(\frac{d N}{d y}\right)_{y=0}^{A A}=a_{A}(\sqrt{s})^{0.3}+b_{A}
$$

and

$$
\left(\frac{d N}{d y}\right)_{y=0}^{p p}=a_{p}(\sqrt{s})^{0.22}+b_{p}
$$

with $a_{A}=0.7613, b_{A}=0.0534 ; a_{p}=0.797 ; b_{p}=0.04123$. We can use these results to obtain the initial entropy density $s_{0}(s)$ in eq. (6) as function of the collision energy. Next we then use eq's. (4), (5) and (6) to express the strangeness suppression factor $\gamma_{s}$ as function of the initial entropy density of the medium produced in the corresponding collision. For the relevant parameters in eq. (6) we take $R_{p p}=0.8 \mathrm{fm}$ for $p p, R_{p P b}=R_{p p}\left(0.5 \bar{N}_{\text {part }}\right)^{1 / 3}$ with $\bar{N}_{\text {part }}=8$ for $p P b$ [12], and $R_{A A}=1.25 A^{1 / 3} \mathrm{fm}$ for $A A$. For the equilibration time, we follow the common choice $\tau_{0}=1 \mathrm{fm}$. The result is shown in Fig. 5 .

We see that we now indeed obtain a universal form of strangeness suppression, in which the $p p$ and $A A$ interpolation curves coincide and with which $p p, p A$ and $A A$ data agree. The relevant variable for the amount of strangeness suppression is thus the initial entropy density. As already indicated above, the pronounced strangeness suppression observed in $p p$ collisions at lower entropy densities disppears when collision energy and hence $s_{0}$ increase. Such an effect had been predicted some time ago as a consequence of the increasing life-time of the thermal medium with increasing collision energy $[6,8]$.

Since finite temperature lattice QCD calculations [13] provide us with the dependence of $s_{0}$ on the initial temperature $T$, we can use the results of Fig. 5 to express $\gamma_{s}$ as a function of $T$. The resulting universal strangeness suppresson as function of the initial temperature is shown in Fig. 6. Here we should keep in mind that the lattice results hold for small values of the baryochemical potential $\mu$, so that the $A A$ points at lowest temperatures (AGS) are to be considered estimates only.

From Fig. 6 it is evident that if the initial temperature of the thermal medium produced in the collision lies below the deconfinement temperature, i.e., if the medium is in a hadronic state, there is strangeness suppression, with $\gamma_{s} \simeq 0.5$. For media of initial temperatures above the transition region, such a suppression is no longer present; it disappears in a very narrow temperature range around 


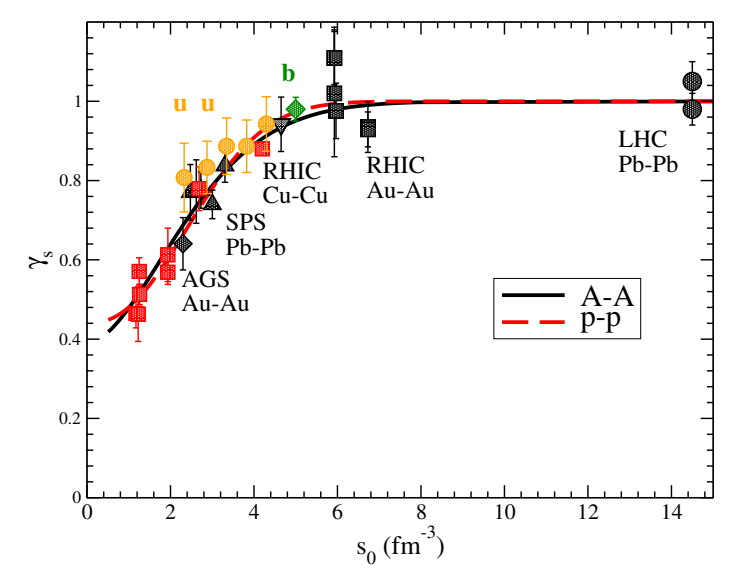

Figure 5. Strangeness suppression $\gamma_{s}\left(s_{0}\right)$ as function of the initial entropy density $s_{0}$.

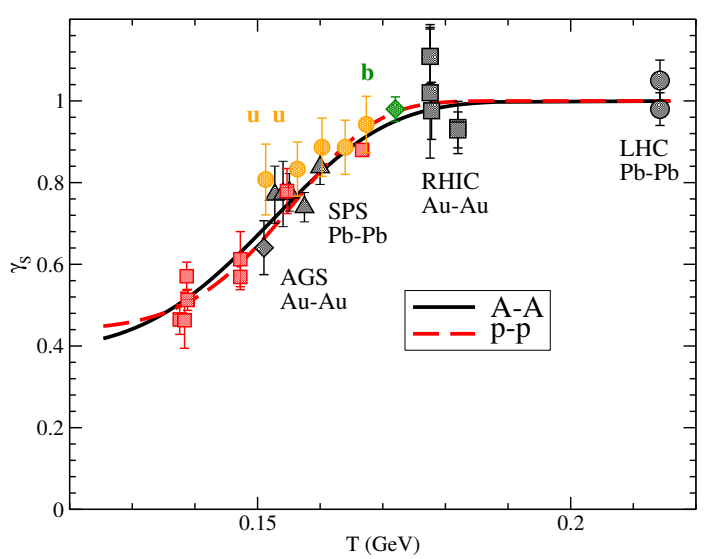

Figure 6. Strangeness suppression $\gamma_{s}(T)$ as function of the initial temperature $T$.

$T_{c} \simeq 160 \mathrm{MeV}$, for $p p$ as well as for nuclear collisions. In other words, strangeness suppression disappears with deconfinement.The same relation to the confinement/deconfinement point is of course also present in Fig. 5: here the transition occurs in a narrow band around the corresponding entropy density at the transition point, $s_{0} \simeq 3 \mathrm{fm}^{-3}$.

To obtain the universal form of strangeness suppression, we had used the average multiplicities. We could, however, use the above formalism also to derive predictions for $\gamma_{s}(s)$ at a fixed cms energy as function of the associated multiplicity. In particular, we could in this way obtain the multiplicity dependence of strangeness suppression in $p p$ collisions at the LHC. This, however, requires as input the transverse area, which also depends on the corresponding final multiplicity, since high multiplicity events arise from large transverse area fluctuations. This has been estimated in specific approaches, such as the color glass condensate [14], but as such remains model-dependent. We therefore postpone such an analysis to later work.

In conclusion, we have shown that in terms of thermal variables, strangeness suppression is a universal phenomenon in all high energy hadronic interactions, $p p, p A$ and $A A$. It decreases with increasing initial entropy density of the produced thermal medium, or equivalently, with increasing 
initial temperature, and it vanishes with the onset of deconfinement. Above the deconfinement transition regime, full equilibration of strange as well as non-strange secondaries is reached for all high energy multihadron production.

\section{References}

[1] J. Rafelski and B. Müller, Phys. Rev. Lett. 48, 1066 and erratum ...(1982).

[2] J. Rafelski, Nucl. Phys. A 418, 215 (1984).

[3] J. S. Hamieh, K. Redlich and A. Tounsi, Phys. Lett. B 486, 61 (2000).

[4] For further references to early work, see

P. Braun-Munzinger, K. Redlich and J. Stachel, in Quark-Gluon Plasma 3, R. C. Hwa and X.-N Wang (Eds.), World Scientific, Singapore 2003.

[5] P. Castorina and H. Satz, Int. J. Mod. Phys. E 23, 1450019 (2014).

[6] P. Castorina, S. Plumari and H. Satz, J. Mod. Phys. E 25, 1650058 (2016).

[7] J. Letessier, J. Rafelski and A. Tounsi, Phys. Rev. C 64,406 (1994).

[8] P. Castorina and H. Satz, Eur. Phys. J. CA 52, 200 (2016).

[9] F. Becattini et al., Eur. Phys. J. C56, 493 (2008).

F. Becattini and R. Fries, Landolt-Börnstein 23, 208 (2010).

F. Becattini, J. Manninen and M. Gazdzicki, Phys. Rev. C 73, 044905 (2006).

F. Becattini and J. Manninen, Phys. Rev. C 78, 054901 (2008)

[10] The $\gamma_{s}$ values were obtained in the following data analyses and in ref. [9], where also the original experimental references are given:

F. Becattini et al. Eur. Phys. J. C66, 377 (2010).

M. Floris et al., Nucl. Phys. A 931, 103 (2014).

F. Becattini, hep-ph/9701275.

F. Becattini et al., Phys. Rev. Lett. 111, 082302 (2013).

The fits eq's (4) and (5) were made by S.Plumari.

[11] K. Aamodt et al. (ALICE Coll.), Phys. Rev. Lett. 105, 252301 (2010).

[12] B. Abelev et al. (ALICE Coll.), Phys. Rev. Lett. 110, 052301 (2013).

[13] A. Bazazov et al. (HotQCD), Phys. Rev. D 90, 094503 (2014).

[14] L. McLerran, M. Praszalowicz and B. Schenke, Nucl. Phys. A 916, 210 (2013);

L. McLerran and P. Tribedy, Nucl. Phys. A 945, 216 (2016). 